\title{
Adiabatic Models of the Efimov Effect
}

\author{
Y. Abe, B.G. Giraud, Yukap Hahn, \\ H. Inamori, and J. LeTourneux
}

CRM-2562

1997

Yukawa Institute for Theoretical Physics, Kyoto 606, Japan

Service de Physique Théorique, DSM, CE Saclay, F-91191 Gif/Yvette, France

Physics Dept, University of Connecticut, Storrs, CT, 06269, USA

Ecole Centrale, F-92295 Châtenay-Malabry Cedex, France

Département de physique and Centre de recherches mathématiques, Université de Montréal, Montréal, P.Q. H3C 3J7 Canada 



\begin{abstract}
Elementary demonstrations of the 3-body Efimov effect are provided for several models involving a light particle and two heavy ones, and differing by the nature of the 2-body (local or non-local) potentials which bind at zero energy the light particle to each of the heavy ones. The proofs rely on Born-Oppenheimer approximations, which are actually projections of the Hamiltonian into suitable subspaces, and they use the correct reduced mass. A numerical estimate of the long range potential responsible for the accumulation of Efimov bound states is obtained through Padé approximants.
\end{abstract}

\title{
1. Introduction
}

As claimed by Efimov ${ }^{1)}$, an arbitrarily large number of bound states is induced in the quantum 3-body problem by 2-body forces which marginally bind pairs of particles. This claim was studied by several authors ${ }^{2-5)}$. In particular, it was validated by a formal proof ${ }^{2}$. It is often difficult, however, to develop a physical intuition of this Efimov phenomenon from analyses using Faddeev equations and/or hyperspherical coordinates. One can reach a better physical understanding by considering the situation where one particle of mass $m$ is much lighter than the other two, of masses $M$ and $M^{\prime}$. Indeed, one can then identify "fast" and "slow" degrees of freedom, which in the following will be identified by $\vec{y}$ and $\vec{x}$, respectively. In the Born-Oppenheimer (BO) approximation, the evolution of $\vec{y}$ is driven by a "fast Hamiltonian" $H_{f}$. The lowest eigenvalue of the latter, $\eta(x)$, which is parametrized by the "slow" degree of freedom, finally becomes an effective potential in the "slow Hamiltonian" $H_{s}$ which drives $\vec{x}$. The fact that $\eta$ turns out to be long ranged, $\eta \propto-1 / x^{2}$, induces the expected accumulation of 3-body bound states. The present paper studies the Efimov effect through the general existence, or possible absence, of such an effective potential $\eta \propto-1 / x^{2}$ in adiabatic models.

This adiabatic situation was mentioned by Efimov himself, and it also underlies the exactly soluble models of Fonseca, Redish and Shanley ${ }^{3)}$, and Giraud and $\mathrm{Hahn}^{5)}$. For simplicity, such models use equal masses, $M=M^{\prime}$, for the heavy particles, say 1 and 2. They are thus naturally formulated in terms of the set of Jacobi coordinates, $\vec{x}=\vec{r}_{2}-\vec{r}_{1}, \vec{y}=\vec{r}_{3}-\left(\vec{r}_{1}+\vec{r}_{2}\right) / 2$, and $H_{f}$ contains the reduced mass $\mu_{3}=2 M m /(2 M+m)$, a 3 -body reduced mass slightly different from the 2-body reduced mass $\mu_{2}=M m /(M+m)$ which controls the definition of pair binding at zero energy. Furthermore, such models use separable potentials, which may give rise to problems when a BO freezing of $\vec{x}$ is implemented. Indeed, consider for instance a separable potential for the pair 31, with matrix elements

$$
\left.<\overrightarrow{r_{1}^{\prime}} \overrightarrow{r_{2}^{\prime}} \overrightarrow{r_{3}^{\prime}}|v| \overrightarrow{r_{1}} \overrightarrow{r_{2}} \overrightarrow{r_{3}}\right\rangle=f\left(\overrightarrow{r_{3}^{\prime}}-\overrightarrow{r_{1}^{\prime}}\right) f\left(\overrightarrow{r_{3}}-\overrightarrow{r_{1}}\right) \delta\left[\frac{m \overrightarrow{r_{3}^{\prime}}+M \overrightarrow{r_{1}^{\prime}}-m \overrightarrow{r_{3}}-M \overrightarrow{r_{1}}}{M+m}\right] \delta\left(\overrightarrow{r_{2}^{\prime}}-\overrightarrow{r_{2}}\right) .
$$

In terms of the total center-of-mass coordinate $\vec{R}$ and the set of Jacobi coordinates $\vec{x}, \vec{y}$ this becomes,

$$
<\overrightarrow{y^{\prime}} \overrightarrow{x^{\prime}} \overrightarrow{R^{\prime}}|v| \vec{y} \vec{x} \vec{R}>=f\left(\overrightarrow{y^{\prime}}+\overrightarrow{x^{\prime}} / 2\right) f(\vec{y}+\vec{x} / 2) \delta\left[\frac{(2 M+m)\left(\overrightarrow{x^{\prime}}-\vec{x}\right)-2 m\left(\overrightarrow{y^{\prime}}-\vec{y}\right)}{2(M+m)}\right] \delta\left(\overrightarrow{R^{\prime}}-\vec{R}\right) .
$$

The mixture of $\vec{x}, \overrightarrow{x^{\prime}}, \vec{y}$ and $\overrightarrow{y^{\prime}}$ in the delta-function $\delta\left(\left[(2 M+m)\left(\overrightarrow{x^{\prime}}-\vec{x}\right)-2 m\left(\overrightarrow{y^{\prime}}-\vec{y}\right)\right] /[2(M+m)]\right)$ clearly makes it difficult to freeze $v$ into a separable potential $f\left(\overrightarrow{y^{\prime}}+\vec{x} / 2\right) f(\vec{y}+\vec{x} / 2)$ parametrized by $\vec{x}$ only and acting upon $\vec{y}$ within the framework of $H_{f}$. These two difficulties, namely the reduced mass inconsistency and the problem of correctly defining adiabatic freezing in Jacobi coordinates for non-local potentials, are the main motivations of this paper.

In Section 2 we briefly recall the whole argument which relates the BO approximation and the generator coordinate method to "proofs" of the Efimov effect. Then, focusing our discussion on $H_{f}$, we introduce three models suitable for the emergence of an effective potential $\eta \propto-1 / x^{2}$. These three models use, respectively, two separable, then one separable and one local, and finally two local potentials. Their properties are investigated in Section 3. A numerical estimation of the effective BO potential $\eta$ is presented in Section 4, where Padé approximants provide an upper and a lower bound of $\eta$. Finally, Section 5 contains our discussion and conclusion. 
2. Adiabaticity, projection into subspaces and soluble models

Consider the following partition of the Hamiltonian in the $(\vec{x}, \vec{y})$ representation,

$$
H=H_{s}+H_{f}, \quad H_{s}=P^{2} / M+p^{2} /\left(2 \mu_{3}\right)-p^{2} /\left(2 \mu_{2}\right), H_{f}=p^{2} /\left(2 \mu_{2}\right)-V(|\vec{x} / 2+\vec{y}|)-V^{\prime}(|-\vec{x} / 2+\vec{y}|),
$$

where the short-range potential $v_{12}(x)$ has been omitted, since it is essentially irrelevant for our argument concerning behaviors at large values of $x$. For the sake of simplicity, the masses of particles 1 and 2 were set to be equal, but the corresponding scalar potentials $V \equiv-v_{13}$ and $V^{\prime} \equiv-v_{23}$ may differ from each other. The coordinates $\vec{x}=\vec{r}_{2}-\vec{r}_{1}$, $\vec{y}=\vec{r}_{3}-\left(\vec{r}_{1}+\vec{r}_{2}\right) / 2$ still refer to the partition (12)3. The operators $\vec{P}, \vec{p}$ are the corresponding momenta. The correct relative mass $\mu_{2}$, the one that is involved when determining the critical strengths of $V$ and $V^{\prime}$, occurs in $H_{f}, \operatorname{Eq}(2.1)$. Hence, the problem created by $\mu_{3}$ and noticed in ref. ${ }^{5}$ ) does not creep any more at this level of the theory. However, it is carried over to $H_{s}$, naturally, trough the corrective terms $\frac{1}{2} p^{2}\left(1 / \mu_{3}-1 / \mu_{2}\right)$. Since $1 / \mu_{3}-1 / \mu_{2}=-1 /(2 M)$, it can be expected that

$$
H_{s}=P^{2} / M-p^{2} /(4 M),
$$

because of its global 1/M coefficient, is still a "slow" Hamiltonian when compared with $H_{f}$.

For the sake of simplicity, all particles are assumed to be spinless and the attractive nature of the interactions is stressed in Eq.(2.1) via $i$ ) explicit minus signs, ii) a choice of $-v_{31}$ and $-v_{32}$ as semi-definite, positive operators $V$, $V^{\prime}$, respectively, and iii) positive, implicit coupling constants $\lambda, \lambda^{\prime}$. The system of units and parameters in this paper is such that $2 \mu_{2}=1, \hbar=1$, and the ranges $\gamma$ of all interactions, form factors, etc. are also set to the value $\gamma=1$. Finally, in numerical applications, the value $\varepsilon \equiv m / M=0.1$ will be regarded as a reasonable order of magnitude for the adiabaticity parameter.

The exact natures of $V, V^{\prime}$ and the values of their implicit coupling constants $\lambda, \lambda^{\prime}$ are such that the two-body Hamiltonians $p^{2}-V$ and $p^{2}-V^{\prime}$ have just a zero energy "bound" state, $\chi_{0}$ and $\chi_{0}^{\prime}$, respectively.

In the generalized version of the Born-Oppenheimer method that was suggested in ref. ${ }^{5)}$, the dynamics is first projected into a subspace of constrained wave functions, where $\vec{x}$ takes, on the average and up to small fluctuations, an arbitrary predefined value $\vec{\xi}$. Such constrained states read, for instance,

$$
\vec{\xi}(\vec{x}, \vec{y})=\pi^{-3 / 4} \ell^{-3 / 2} \exp \left[-(\vec{x}-\vec{\xi})^{2} /\left(2 \ell^{2}\right)\right] \quad \chi(\vec{y}),
$$

where $\ell$ controls the fluctuations of $\vec{x}$ around $\vec{\xi}$. In the limit $\ell \rightarrow 0$, one recovers the usual BO ansatz,

$$
\vec{\xi}(\vec{x}, \vec{y}) \propto \delta(\vec{x}-\vec{\xi}) \chi(\vec{y}),
$$

where $\vec{x}$ is strictly frozen. In any case, in the spirit of the BO method, one minimizes $<_{\vec{\xi}}\left|H_{f}\right|_{\vec{\xi}}>/ \vec{\xi}_{\vec{\xi}} \mid \vec{\xi}>$ with respect to variations of $\chi$. That resulting $\chi$, which induces a minimum of such a "fast" energy, obviously depends on the constraint $\vec{\xi}$ and may be labelled $\chi_{\vec{\xi}}$.

Whether $\ell$ is finite or vanishing, the factorization of as a product of a wave packet or $\delta$-function for $\vec{x}$ and a trial function for $\vec{y}$ is a Hartree-like ansatz. Hence the variational minimization of $\left\langle_{\vec{\xi}}\left|H_{f}\right|_{\vec{\xi}}\right\rangle /\left\langle_{\vec{\xi}} \mid \vec{\xi}\right\rangle$ results at once into a diagonalization problem for $\chi$.

In the case of local potentials the simplest way to implement the freezing process obviously corresponds to Eq.(2.4), when $\vec{x}$ is replaced by a simple parameter $\vec{\xi}$ in the potentials $V$ and $V^{\prime}$. This yields a typical BO fast equation,

$$
\left[p^{2}-\mathcal{V}-\mathcal{V}^{\prime}+\omega^{2}\right] \mid \chi>=0
$$

where $\mathcal{V}$ and $\mathcal{V}^{\prime}$ are the operators deduced from $V$ and $V^{\prime}$ when $\vec{x}$ is frozen at the value $\vec{\xi}$, and we have defined, for the sake of convenience, $\omega^{2} \equiv-\eta$.

Non-local potentials also induce an equation of the form of (2.5), but $\mathcal{V}$ and $\mathcal{V}^{\prime}$ are then Hartree potentials and $H_{f}$ is a Hartree Hamiltonian. For separable potentials, for instance, see Eq.(1.2), the $\vec{x}$-integration takes care, as a consequence of the Gaussian in Eq.(2.3), of the difficulties arising from $\delta$-functions such as the one appearing in Eq.(1.2), $\delta\left(\left[(2 M+m)\left(\overrightarrow{x^{\prime}}-\vec{x}\right)-2 m\left(\overrightarrow{y^{\prime}}-\vec{y}\right)\right] /[2(M+m)]\right)$. It is instructive to consider the special case where the form factor $f$ of a separable potential is a Gaussian of width $L$, for instance,

$<\vec{y}^{\prime}|\mathcal{V}| \vec{y}>\propto \int d \vec{x}^{\prime} d \vec{x} \exp \left[-\frac{\left(\vec{x}^{\prime}-\vec{\xi}\right)^{2}+(\vec{x}-\vec{\xi})^{2}}{2 \ell^{2}}-\frac{\left(\vec{y}^{\prime}-\vec{x}^{\prime} / 2\right)^{2}+(\vec{y}-\vec{x} / 2)^{2}}{2 L^{2}}\right] \delta\left[\frac{(1+\varepsilon / 2)\left(\vec{x}^{\prime}-\vec{x}\right)-\varepsilon\left(\vec{y}^{\prime}-\vec{y}\right)}{1+\varepsilon}\right]$. 
The integration $\int d \vec{x}^{\prime} d \vec{x}$ can easily be performed as $\int d \vec{S} \int d\left(\vec{x}^{\prime}-\vec{x}\right)$, where $\vec{S}$ stands for $\left(\vec{x}^{\prime}+\vec{x}\right) / 2$, and one obtains,

$$
<\vec{y}^{\prime}|\mathcal{V}| \vec{y}>\propto \mathcal{K} \int d \vec{S} \exp \left[-\frac{(\vec{S}-\vec{\xi})^{2}}{\ell^{2}}-\frac{\left(\vec{y}^{\prime}+\vec{y}-\vec{S}\right)^{2}}{4 L^{2}}\right],
$$

with an inessential numerical factor, and

$$
\mathcal{K}=\exp \left[-\frac{\varepsilon^{2}\left(\vec{y}^{\prime}-\vec{y}\right)^{2}}{4(1+\varepsilon / 2)^{2} \ell^{2}}\right] \exp \left[-\frac{\left(\vec{y}^{\prime}-\vec{y}\right)^{2}}{4(1+\varepsilon / 2)^{2} L^{2}}\right]
$$

It is easy to verify that, when $\varepsilon<<\ell / L<<1$, this boils down to $<\vec{y}^{\prime}|\mathcal{V}| \vec{y}>\propto \exp \left(-\left[\left(\vec{y}^{\prime}-\vec{\xi} / 2\right)^{2}+(\vec{y}-\vec{\xi} / 2)^{2}\right] /\left(2 L^{2}\right)\right)$, the expected, separable result. It can be concluded that the freezing may distort non locality properties mildly only, with corrections of first order, at most, in the adiabaticity parameter.

For the sake of completeness we recall that, once the functions $\chi_{\vec{\xi}}(\vec{y})$ have been calculated for all $\vec{\xi}$, the full ansatz for the 3-body wave function uses $\vec{\xi}$ as a generator coordinate,

$$
(\vec{x}, \vec{y})=\int d \vec{\xi} \varphi(\vec{\xi}) \vec{\xi}(\vec{x}, \vec{y})
$$

with the transparent special case derived from Eq.(2.4),

$$
(\vec{x}, \vec{y})=\varphi(\vec{x}) \chi_{\vec{x}}(\vec{y}) .
$$

The exact form of the Griffin-Hill-Wheeler equation which must then be solved to obtain $\varphi(\vec{\xi})$ is an obvious generalization of the usual BO "slow" equation. This form depends on the properties of the kernel $\mathcal{H}\left(\vec{\xi}, \overrightarrow{\xi^{\prime}}\right) \equiv<\vec{\xi}|H|_{\vec{\xi}^{\prime}}>$, the diagonal of which obviously includes the eigenvalue $\eta$. In so far as $\eta$ behaves as a long range potential when $\xi \rightarrow \infty$, the slow equation is thus expected to contain an Efimov effect. We now postpone the study of the "slow" equation and, in this paper, for the sake of simplicity, we consider Eq.(2.5) only, through several models. We concentrate on the behavior of $\eta$, or $\omega$ as well, when $\xi \rightarrow \infty$.

The soluble models of Eq.(2.5) that we investigate are :

Model 1 : Two separable potentials, namely $\mathcal{V} \propto|f><f|$ and $\mathcal{V}^{\prime} \propto\left|f^{\prime}><f^{\prime}\right|$, where the form factors $f$ and $f^{\prime}$ have the shape of Yukawa potentials and read, in momentum representation, $f(\vec{p})=1 /\left[\pi\left(p^{2}+\gamma^{2}\right)\right]$, and $f^{\prime}(\vec{p})=1 /\left[\pi\left(p^{2}+\gamma^{\prime 2}\right)\right]$, respectively. With $\mathcal{V}^{\prime}$ comes the operator $T$ which implements a translation through a distance $\vec{\xi}$. (It makes no difference whether $\mathcal{V}$ is centered at $y=0$ and $\mathcal{V}^{\prime}$ is centered at $\vec{\xi}$ or such potentials are centered at $\pm \vec{\xi} / 2$.) Hence $<\vec{p}|T| f^{\prime}>=\exp (-i \vec{p} \cdot \vec{\xi}) /\left[\pi\left(p^{2}+\gamma^{\prime 2}\right)\right]$. This model differs by small details only (the reduced mass, the breaking of symmetry) from the ones studied in refs. ${ }^{3,5)}$;

Model $2: \mathcal{V}$ is the same, but now $\mathcal{V}^{\prime}$ is local, which facilitates its BO freezing. For the sake of definiteness, we set $\mathcal{V}^{\prime}(\vec{y}) \propto \theta\left(\gamma^{\prime}-y\right)$, a square well of radius $\gamma^{\prime}$ and depth 1. This comes into Eq.(2.5) under the translated form $T \mathcal{V}^{\prime} T^{\dagger}$, naturally;

Model 3 : Both $\mathcal{V}$ and $\mathcal{V}^{\prime}$ are local. For the sake of simplicity, they are taken to be square wells with ranges $\gamma$ and $\gamma^{\prime}$, respectively.

In the next Section, we show how, in these three cases, elementary manipulations of Eq.(2.5) confirm that $\omega \propto 1 / \xi$.

\section{Asymptotic behavior of the eigenvalue of the fast $\mathrm{BO}$ equation}

Model 1 :

In this case, Eq.(2.5) can be written as

$$
\left(p^{2}+\omega^{2}-\lambda^{\prime} T\left|f^{\prime}><f^{\prime}\right| T^{\dagger}\right)|\chi>=\lambda| f><f \mid \chi>,
$$

and it is convenient to define the resolvent $G=\left(p^{2}+\omega^{2}-\lambda^{\prime}\left|f^{\prime}><f^{\prime}\right|\right)^{-1}$ and the bare resolvent $g=\left(p^{2}+\omega^{2}\right)^{-1}$. For our Yukawa form factors, the coupling constants suitable for zero energy binding are $\lambda=[\langle f|g(\omega=0)| f\rangle]^{-1}=\gamma^{3}$ and, similarly, $\lambda^{\prime}=\gamma^{\prime 3}$. 
Since $G$ has no pole as long as $\omega \neq 0$, one can easily express $\mid \chi>$ as $\left|\chi>=\lambda T G T^{\dagger}\right| f><f \mid \chi>$, and, finally, the quantization condition which relates $\omega$ to the parameter $\vec{\xi}$ of $T$, reads

$$
\frac{1}{\gamma^{3}}=<f\left|T G T^{\dagger}\right| f>
$$

Using the perturbative expansion of $G$ in terms of $\lambda^{\prime}$,

$$
G=g+\lambda^{\prime} g\left|f^{\prime}><f^{\prime}\right| g+\lambda^{\prime 2} g\left|f^{\prime}><f^{\prime}\right| g\left|f^{\prime}><f^{\prime}\right| g+\ldots
$$

one can reformulate this condition as

$$
\frac{1}{\gamma^{3}}-<f|g| f>=<f|g T| f^{\prime}>\lambda^{\prime}\left[1-\lambda^{\prime}<f^{\prime}|g| f^{\prime}>\right]^{-1}<f^{\prime}\left|T^{\dagger} g\right| f>.
$$

and, more explicitly, as

$$
\frac{1}{\gamma^{3}}-\frac{1}{\gamma(\gamma+\omega)^{2}}=|<f| g T\left|f^{\prime}>\right|^{2}\left[\frac{1}{\lambda^{\prime}}-<f^{\prime}|g| f^{\prime}>\right]^{-1}=|<f| g T\left|f^{\prime}>\right|^{2}\left[\frac{1}{\gamma^{\prime 3}}-\frac{1}{\gamma^{\prime}\left(\gamma^{\prime}+\omega\right)^{2}}\right]^{-1} .
$$

An elementary though slightly tedious calculation of the numerator $|<f| g T\left|f^{\prime}>\right|^{2}$ of the right-hand side shows that it is dominated by a (scalar) term of the form $\exp (-2 \omega \xi) / \xi^{2}$ when $\xi \rightarrow \infty$. This behavior is actually easy to understand. Indeed, in the coordinate representation, the bare propagator $g$ has a Yukawa form in terms of the distance between its arguments, $<\vec{y}|g| \vec{y}^{\prime}>\propto \exp \left(-\omega\left|\vec{y}-\vec{y}^{\prime}\right|\right) /\left|\vec{y}-\vec{y}^{\prime}\right|$, while $f$ and $T f^{\prime}$ are localised form factors, separated by a distance $\xi$ through the translation operator $T$. The same translation will intervene when computing $<f|g T| f^{\prime}>$, since $\left\langle f|g| \vec{y}>=[\exp (-\omega y)-\exp (-\gamma y)] /\left[(2 \pi)^{\frac{1}{2}}\left(\gamma^{2}-\omega^{2}\right) y\right]\right.$. The equation (3.4) then boils down to $\omega \simeq K \exp (-2 \omega \xi) /\left(\xi^{2} \omega\right)$, where $K$ is a constant. This transcendental equation has the solution $\omega=\mathcal{C} / \xi$, where the constant $\mathcal{C}$ should obey the condition $\mathcal{C}=K^{\frac{1}{2}} \exp (-\mathcal{C})$. Hence the expected result $\omega \propto 1 / \xi$, and an effective potential $\eta \propto-\mathcal{C}^{2} / x^{2}$ for the slow BO equation. It will be noticed that the coefficient $\mathcal{C}$ is a finite number, even when $\varepsilon \rightarrow 0$.

\section{Model 2:}

Now, Eq.(2.5) can be written in the form

$$
\left(p^{2}+\omega^{2}-\lambda^{\prime} T \mathcal{V}^{\prime} T^{\dagger}\right)|\chi>=\lambda| f><f \mid \chi>,
$$

and while the bare resolvent $g=\left(p^{2}+\omega^{2}\right)^{-1}$ remains the same, it is convenient to define $G=\left(p^{2}+\omega^{2}-\lambda^{\prime} \mathcal{V}^{\prime}\right)^{-1}$. Since we have chosen $\mathcal{V}^{\prime}$ to be a square well of radius $\gamma^{\prime}=1$, there will be binding at zero energy if $\lambda^{\prime}=\pi^{2} / 4$. One finds again the solution $\left.\left|\chi>=\lambda T G T^{\dagger}\right| f\right\rangle\langle f| \chi>$, and, again, the relation between $\omega$ and $\vec{\xi}$ is given by Eq.(3.2).The same perturbative expansion of $G$ in terms of $\lambda^{\prime}$ reads now,

$$
G=g+\lambda^{\prime} g \mathcal{V}^{\prime} g+\lambda^{\prime 2} g \mathcal{V}^{\prime} g \mathcal{V}^{\prime} g+\ldots
$$

and can be rewritten, in terms of the operator square root $W^{\prime} \equiv \mathcal{V}^{\prime \frac{1}{2}}$, as

$$
G-g=\lambda^{\prime} g W^{\prime}\left[1-\lambda^{\prime} W^{\prime} g W^{\prime}\right]^{-1} W^{\prime} g .
$$

Notice, incidentally, that for square wells $\mathcal{V}^{\prime}$ of depth 1, one has $\mathcal{V}^{\prime \frac{1}{2}}=\mathcal{V}^{\prime}$. All told, Eq.(3.5) becomes

$$
\frac{1}{\lambda}-\frac{1}{\gamma(\gamma+\omega)^{2}}=<f\left|g T W^{\prime}\left[\frac{1}{\lambda^{\prime}}-W^{\prime} g W^{\prime}\right]^{-1} W^{\prime} T^{\dagger} g\right| f>.
$$

The left-hand side of this relation is identical to that of the previous similar relation, Eq.(3.4). Their right-hand sides are also very similar. Indeed, on the one hand, the same separation distance $\xi$ occurs between $f$ and $W^{\prime}$ in Eq.(3.7) as between $f$ and $f^{\prime}$ in the term $<f|g T| f^{\prime}>$ of Eq.(3.4). The square of the same propagation damping factor $\exp (-\omega \xi) / \xi$, is thus present. On the other hand, while the denominator $1 / \lambda^{\prime}-<f^{\prime}|g| f^{\prime}>$ of Eq.(3.4) provides an explicit $\omega$ factor, this right-hand side $\omega^{-1}$ factor is only implicit in Eq.(3.7), through the propagator $\left[1 / \lambda^{\prime}-W^{\prime} g W^{\prime}\right]^{-1}$.

To make the factor in question more explicit, consider the eigenstate ${ }_{\omega}^{\prime}$ of $W^{\prime} g W^{\prime}$ corresponding to its largest eigenvalue $\omega_{\omega}$, namely that eigenvalue which reaches the value $1 / \lambda^{\prime}$ when $\omega \rightarrow 0$. (We recall here that when $\omega$ increases from 0 to $\infty$, the operator $W^{\prime} g(\omega) W^{\prime}$, which is square integrable and positive semi-definite, decreases monotonically, 
with all its eigenvalues discrete and decreasing.) The eigenstate ${ }_{\omega}^{\prime}$ is square integrable, like the other eigenstates of $W^{\prime} g W^{\prime}$. Let $Q \equiv 1-\left.\right|_{\omega} ^{\prime}><{ }_{\omega}^{\prime} \mid$ be the usual projector orthogonal to ${ }_{\omega}^{\prime}$, normalized to unity. Then Eq.(3.7) reads,

$$
\frac{2 \gamma \omega+\omega^{2}}{\gamma^{3}(\gamma+\omega)^{2}}-\frac{\left.|<f| g T W^{\prime}\right|_{\omega} ^{\prime}>\left.\right|^{2}}{1 / \lambda^{\prime}-\omega}=<f\left|g T W^{\prime} Q\left[\frac{1}{\lambda^{\prime}}-Q W^{\prime} g W^{\prime} Q\right]^{-1} Q W^{\prime} T^{\dagger} g\right| f>,
$$

the right-hand side of which is regular for all values of $\omega \geq 0$. We show in the Appendix that the derivative $d / d \omega$ has a finite, negative limit $-\sigma_{0}$ when $\omega \rightarrow 0$. Then, with $\gamma=1$ and $\omega \rightarrow 0$, Eq.(3.8) boils down to

$$
2 \omega^{2}-\frac{\left.|<f| g T W^{\prime}\right|_{\omega} ^{\prime}>\left.\right|^{2}}{\sigma_{0}}=\omega<f\left|g T W^{\prime} Q\left[\frac{1}{\lambda^{\prime}}-Q W^{\prime} g W^{\prime} Q\right]^{-1} Q W^{\prime} T^{\dagger} g\right| f>.
$$

All overlap matrix elements being dominated by an asymptotic decrease of the form $\exp (-\omega \xi) / \xi$, the ansatz $\omega \simeq \mathcal{C} / \xi$ dwarfs the right-hand side of $\mathrm{Eq}(3.9)$. This ansatz, with a suitable value of $\mathcal{C}$, satisfies the residual condition $2 \omega^{2}=\left.|<f| g T W^{\prime}\right|_{0}{ }^{\prime}>\left.\right|^{2} / \sigma_{0}$, where ${ }_{0}{ }^{\prime}$ is the limit of ${ }_{\omega}^{\prime}$, namely the product of $W^{\prime}$ with the zero energy (quasi) bound state of $p^{2}+\lambda^{\prime} \mathcal{V}^{\prime}$. (This product is here square normalized to unity.) It will be noticed that here again the coefficient $\mathcal{C}$ remains a finite number when $\varepsilon \rightarrow 0$.

\section{Model 3:}

Both potentials being local, there is no difficulty in the freezing of $\vec{x}$ at a parameter value $\vec{\xi}$. For the sake of conciseness and simplicity, we include the translation operators in the definition of $\mathcal{V}^{\prime}$ and set $\mathcal{V}^{\prime}=T \mathcal{V} T^{\dagger}$. Simultaneously, we now include $\lambda$ and $\lambda^{\prime}$ in the definitions of $\mathcal{V}$ and $\mathcal{V}^{\prime}$, respectively. Their eigenvalues are multiplied in the same way. The corresponding square root operators $W$ and $W^{\prime}$ are multiplied by $\lambda^{\frac{1}{2}}$ and $\lambda^{\frac{1}{2}}$, accordingly. It is then convenient to define the operators $A=W g W, A^{\prime}=W^{\prime} g W^{\prime}$ and $B=W g W^{\prime}$, which are parametrized by $\omega$. Respectively, the eigenstate of $A\left(A^{\prime}\right)$, with the largest eigenvalue $\left({ }^{\prime}\right)$ will be denoted by $\left({ }^{\prime}\right)$ with a square normalizable limit $0\left({ }_{0}{ }^{\prime}\right)$ when $\omega \rightarrow 0$. In that limit, this largest eigenvalue (') of $A\left(A^{\prime}\right)$ increases and reaches 1 , and we know from the Appendix that both $1-$ and $1-^{\prime}$ are first-order quantities in $\omega$. For all values of $\omega$ the normalization \langle|$>=\left\langle{ }^{\prime} \mid{ }^{\prime}\right\rangle=1$ can be chosen. It will be noticed that because of the implicit translation operators now present in $\mathcal{V}^{\prime}$ and $W^{\prime}$, the states and ' are localised at a distance $\xi$ from each other. The behavior of $B$ as essentially proportional to $\exp (-\omega \xi) / \xi$ is also transparent. Hence, because of the denominator $\xi$, this operator $B$ can be considered as a small operator when $\xi$ is large, whether $\omega$ is fixed or simulateneously decreases like $1 / \xi$. Here "small" refers to the comparison between $B$ and $A\left(A^{\prime}\right)$, the latter having at least one eigenvalue $\left({ }^{\prime}\right)$ close to 1 . Furthermore, the special choice $\mathcal{V}^{\prime}=T \mathcal{V} T^{\dagger}$ makes of $A^{\prime}=T A T^{\dagger}$ a simply translated version of $A$. Hence ' $=$, the spectra of $A$ and $A^{\prime}$ are the same, and their respective eigenstates differ only by the translation induced by $T$.

Now, we duplicate Eq.(2.5) according to

$$
\begin{aligned}
\left(\omega^{2}+p^{2}-\mathcal{V}\right) \mid \chi> & =\mathcal{V}^{\prime} \mid \chi> \\
\left(\omega^{2}+p^{2}-\mathcal{V}^{\prime}\right) \mid \chi> & =\mathcal{V} \mid \chi>
\end{aligned}
$$

or,

$$
\begin{aligned}
& (1-A) W\left|\chi>=B \quad W^{\prime}\right| \chi> \\
& \left(1-A^{\prime}\right) W^{\prime}\left|\chi>=B^{\dagger} W\right| \chi>
\end{aligned}
$$

This reads like a hermitian diagonalization problem with a two-component eigenvector $\mid \zeta, \zeta^{\prime}>\equiv\left(W\left|\chi>, W^{\prime}\right| \chi>\right)$. Clearly, it can be approximated by the "unperturbed" problem $(-A)\left|>=0,\left(-A^{\prime}\right)\right|^{\prime}>=0$, obtained by neglecting $B$, hence approximating $\mid \zeta, \zeta^{\prime}>$ by $2^{-\frac{1}{2}}\left(|>,|^{\prime}>\right)$. The factor $2^{-\frac{1}{2}}$ is introduced here for a suitable normalization of the "unperturbed" state in this two-component representation. Let $\mathcal{Q}$ be the projector orthogonal to this state $2^{-\frac{1}{2}}\left(|>,|^{\prime}>\right)$, and let us denote by $\mathcal{A}$ the diagonal matrix of operators made of $A$ and $A^{\prime}$ in its diagonal. In the same way let $\mathcal{B}$ be the purely off-diagonal matrix of operators $\left(\begin{array}{cc}0 & B \\ B^{\dagger} & 0\end{array}\right)$. The "perturbed" eigenvalue must be 1 , like the "unperturbed" eigenvalue. Hence the Brillouin-Wigner equation for eigenvalues reads

$$
1=+2^{-1}\left[\left(<\left|,<^{\prime}\right|\right) \mathcal{B}\left(|>,|^{\prime}>\right)+\left(<\left|,<^{\prime}\right|\right) \mathcal{B} \frac{\mathcal{Q}}{1-\mathcal{A}-\mathcal{Q B} \mathcal{Q}} \mathcal{B}\left(|>,|{ }^{\prime}>\right)\right] .
$$

This is the condition needed to relate $\omega$ and $\xi$. It simplifies to

$$
1-=2^{-1}\left(<|B|^{\prime}>+<^{\prime}\left|B^{\dagger}\right|>\right)+2^{-1}\left(<^{\prime}\left|B^{\dagger},<\right| B\right) \frac{\mathcal{Q}}{1-\mathcal{A}-\mathcal{Q B \mathcal { Q }}}\left(\left.B\right|^{\prime}>, B^{\dagger} \mid>\right) .
$$


We recall here that and ' are both localised within a finite radius, the former around the origin and the latter at a distance $\xi$ away, because of the factors $W$ and $W^{\prime}$ which they include. Hence the matrix elements $\left\langle|B|{ }^{\prime}>\right.$ and $\left\langle{ }^{\prime}\left|B^{\dagger}\right|>\right.$, which incidentally are equal, are essentially proportional to $\exp (-\omega \xi) / \xi$. This behavior is again due to the product of the translation operator $T$ and the propagator $g$ (see the definition of $B$ ). Furthermore the difference $1-$ is known to be of first order with respect to $\omega$. Finally, because of the projector $\mathcal{Q}$, the last term in the right-hand side of Eq.(13), namely the matrix element of $\mathcal{B} \mathcal{Q}[1-\mathcal{A}-\mathcal{Q B Q}]^{-1} \mathcal{B}$, is regular in the limit $\omega \rightarrow 0$ and its behavior as a second order factor $\exp (-2 \omega \xi) / \xi^{2}$ is obvious. Hence it is made neglegible by the ansatz $\omega \propto \mathcal{C} / \xi$ and Eq.(3.13) boils down to $1-=\left\langle|B|^{\prime}>\right.$, which is again satisfied by this ansatz. QED It will be noticed once more that the coefficient $\mathcal{C}$ is finite, even when $\varepsilon \rightarrow 0$.

To conclude this Section, in three models we have found that whenever the two-body Hamiltonians $p^{2}+\mathcal{V}$ and $p^{2}+\mathcal{V}^{\prime}$ have a zero energy bound state (in $s$-waves for three dimensions), the eigenvalue $-\omega^{2}$ of the Hamiltonian $p^{2}+\mathcal{V}+\mathcal{V}^{\prime}$ is given by a condition, such as Eq.(3.3), Eq.(3.7) or Eq.(3.13), which always boils down to a form $\omega \propto \exp (-\omega \xi) / \xi$, when terms of higher order such as $\omega^{2}$ and $\exp (-2 \omega \xi) / \xi^{2}$ are neglected. The small parameter which defines asymptoticity here is, naturally, the reciprocal $1 / \xi$ of the separation distance $\xi$ between the short range potentials $\mathcal{V}$ and $\mathcal{V}^{\prime}$. Thus the asymptotic behavior $\omega^{2} \propto 1 / \xi^{2}$ seems to occur quite generally.

\section{Numerical application}

We return to model 2 and, more precisely, to Eq.(3.6). Because of the semi-positive nature of the operator $W^{\prime} g W^{\prime}$ it is well known $\left.{ }^{6}\right)$ that Padé approximants of order $[N-1, N]$ and $[N, N]$ with respect to $\lambda^{\prime}$ provide lower and upper bounds, respectively, of the resolvent $\left[1-\lambda^{\prime} W^{\prime} g W^{\prime}\right]^{-1}$. Hence Padé approximants of order $[N, N]$ and $[N+1, N]$ of the whole right-hand side of Eq.(3.7) provide, respectively, an increasing sequence of lower bounds and a decreasing sequence of upper bounds for that right-hand side. Given a value of $\xi$, these bounding sequences of Padé approximants generate in turn systematic lower and upper bounds for $\omega$ via the positions where the graph of $1 / \lambda-<f|g| f\rangle$, plotted as a function of $\omega$, intercepts the graphs of the Padé approximants.

Keeping in mind that $a_{0} \equiv<f|g| f>=1 /(1+\omega)^{2}$, we calculated by brute force the next three perturbative terms, namely, with our choice of form factor, potential shape and parameters,

$$
\begin{aligned}
& a_{1}=\lambda^{\prime}<f\left|g T \mathcal{V}^{\prime} T^{\dagger} g\right| f>=\frac{\pi}{8\left(1-\omega^{2}\right)^{2}} \int d \vec{y} \frac{[\exp (-\omega y)-\exp (-y)]^{2}}{y^{2}} \theta(1-|\vec{y}-\vec{\xi}|), \\
& a_{2}=\lambda^{\prime 2}<f\left|g T \mathcal{V}^{\prime} g \mathcal{V}^{\prime} T^{\dagger} g\right| f>=\pi^{2} /\left[128\left(1-\omega^{2}\right)^{2}\right] \int d \vec{y} d \vec{y}^{\prime} \theta(1-|\vec{y}-\vec{\xi}|) \theta\left(1-\left|\vec{y}^{\prime}-\vec{\xi}\right|\right) \\
& \quad[\exp (-\omega y)-\exp (-y)] \exp \left(-\omega\left|\vec{y}-\vec{y}^{\prime}\right|\right)\left[\exp \left(-\omega y^{\prime}\right)-\exp \left(-y^{\prime}\right)\right] /\left(y\left|\vec{y}-\vec{y}^{\prime}\right| y^{\prime}\right), \\
& a_{3}=\lambda^{\prime 3}<f\left|g T \mathcal{V}^{\prime} g \mathcal{V}^{\prime} g \mathcal{V}^{\prime} T^{\dagger} g\right| f>=\pi^{3} /\left[2048\left(1-\omega^{2}\right)^{2}\right] \int d \vec{y} d \vec{y}^{\prime} d \vec{y}^{\prime} \theta(1-|\vec{y}-\vec{\xi}|) \theta\left(1-\left|\vec{y}^{\prime}-\vec{\xi}\right|\right) \theta\left(1-\left|\vec{y}{ }^{\prime}-\vec{\xi}\right|\right) \\
& \quad[\exp (-\omega y)-\exp (-y)] \exp \left[-\omega\left(\left|\vec{y}-\vec{y}^{\prime}\right|+\left|\vec{y}^{\prime}-\vec{y}{ }^{\prime}\right|\right)\right]\left[\exp \left(-\omega y^{\prime \prime}\right)-\exp \left(-y^{\prime \prime}\right)\right] /\left(y\left|\vec{y}-\vec{y}^{\prime}\right|\left|\vec{y}^{\prime}-\vec{y}{ }^{\prime}\right| y^{\prime \prime}\right),(4.3) c r
\end{aligned}
$$

where actually $\vec{\xi}$ can be oriented along any one of the frame axes, since the result depends on the modulus $\xi$ only. Here the step function $\theta$ accounts for the square well. We estimate the level of numerical accuracy of $a_{3}$ to lie around $1 \%$ when the integration space is sampled with $10^{6}$ points.

The knowledge of $a_{1}$ and $a_{2}$ is sufficient to generate a lower bound of the right-hand side of Eq.(3.7), namely the Padé approximant $[1,1]=a_{1} /\left(1-a_{2} / a_{1}\right)$. With $a_{3}$, an upper bound is in turn provided by the Padé approximant $[2,1]=\left(a_{1}+a_{2}-a_{1} a_{3} / a_{2}\right) /\left(1-a_{3} / a_{2}\right)$. On Fig.1, the plots of $a_{0}+[1,1]$ and $a_{0}+[2,1]$ are shown for $\xi=5$. The latter is indeed above the former. The abscissa scale is that of $\omega \xi$ rather than $\omega$ alone. Both curves intersect the horizontal line with ordinate $1 / \lambda=1$ at positions $\omega \xi \simeq 0.55$ and $\omega \xi \simeq 0.6$, respectively.

Similar plots and the same error bar for the true value of $\omega \xi$ are found for larger values of $\xi$, and even for $\xi \simeq 3,4$, which indicates that the asymptotic regime $\omega \propto 1 / \xi$ starts early. On Fig.2 we show, again in terms of $\omega \xi$, the plots of $a_{0}+[2,1]$ for $\xi=3,4,5,6,7,8$ respectively. The simultaneous crossing of these plots at $\omega \xi \simeq 0.6$ is striking.

A similar property is shown by Fig.3, where the solution of the condition $a_{0}+[1,1]=1$ for again $\xi=3,4,5,6,7,8$ is always $\omega \xi \simeq 0.55$, within the numerical errors to be expected.

In conclusion of this illustrative numerical example, very low order Padé approximants are sufficient to confirm that the eigenvalue $\eta \equiv-\omega^{2}$ of the "fast" BO equation behaves like a long-range potential, $\eta \propto-1 / \xi^{2}$.

5. Discussion and conclusion 
Figure 1. Graphical solution of the conditions $1 / \lambda=a_{0}+[2,1]$ (upper curve) and $1 / \lambda=a_{0}+[1,1]$ (lower curve) for $\xi=5$.

The picture we have been using in this paper is physically transparent : the light particle resonates between the two "critical" potentials centered at the heavy particles. However this picture, a potential model for one particle only, demands at least two justifications, namely

i) an adequate understanding of the freezing of the dynamics with two Jacobi coordinates $\vec{x}, \vec{y}$, into a dynamics for $\vec{y}$ alone,

ii) a proper treatment of such a reduced dynamics.

The first task was accomplished here by projections of the complete dynamics into suitable subspaces, namely 
Figure 2. Plots of $a_{0}+[2,1]$ for $\xi=3,4,5,6,7,8$ in terms of $\omega$, actually scaled to $\omega \xi$ for each value of $\xi$. For $\omega \xi 0.6$ the curves rank in descending order when $\xi$ increases. The converse is true for $\omega \xi 0.6$.

constrained variational subspaces. The projections which we considered include and generalize the Born-Oppenheimer freezing. They are trivial for local potentials. For non-local potentials, however, non-locality properties, separability in particular, may be disturbed. We took great care in verifying that such disturbances can be bounded. Necessary corrections turn out to be of first order in the adiabaticity parameter.

The second task was realized by solving three models which constitute a largely generic class of models. What is striking in these three cases is the similarity of the arguments they generate for the derivation of the asymtotic potential $\eta \equiv-\omega^{2} \propto-1 / \xi^{2}$. One always finds that $\omega$ is asymptotically proportional to the matrix element $\exp (-\omega \xi) / \xi$ 
Figure 3. Same as Fig.2, but now plots of $a_{0}+[1,1]$. For $\omega \xi 0.55$ the curves rank in descending order when $\xi$ increases. The converse occurs for $\omega \xi 0.55$.

which represents the tunneling amplitude between the two resonating potentials. In that sense, this paper gives a strong hint, from very elementary and physical arguments, in favor of the universality of the investigated effect. This should not be too surprising : at large distances $\xi$, the details of the potentials (shapes, values of their short ranges, possible lack of locality, etc.) become irrelevant in the face of the long-range propagation.

Two questions are still pending, however. The first one stems from the fact that this paper investigated only the $\mathrm{BO}$ fast equation, while long range corrections are known ${ }^{5)}$ to be present in the $\mathrm{BO}$ slow equation. It is already obvious from Eq.(2.2), however, that such perturbations, introduced by the slow Hamiltonian, are at most of first 
order in the adiabaticity parameter and can thus be kept under control. This is now under detailed investigation and will form a separate paper. The second question is whether proofs, as elementary as those discussed here, can be found when adiabaticity cannot be invoked. A proper description of both channels where critical potentials are acting may then demand the use of bipolar coordinates, or even of a double set of coordinates, not even necessarily of a Jacobi nature. This is also under investigation.

An additional, technical result obtained in this work deserves to be stressed : Padé approximants of a surprisingly low order are sufficient to probe the validity of the relation $\omega \propto 1 / \chi$.

Acknowledgements : Two authors (YA and HI) thank Service de Physique Théorique, Saclay, for its hospitality during this work. Another author (BG) is grateful to Centre de Recherches Mathématiques, Montréal, for a visit, where the Padé part of this work was started.

\section{References}

1)V.Efimov, Yad.Fiz. 12 (1970) 1080 [Sov.J.Nucl.Phys. 12 (1971) 589]; Phys.Lett. B33 (1970) 563; Nucl.Phys. A210 (1973) 157

2)R.D.Amado and J.V.Noble, Phys.Lett. B35 (1971) 25; Phys.Rev. D5(1972) 1992

3)A.C.Fonseca, E.F.Redish and P.E.Shanley, Nucl.Phys. A320 (1979) 273

4)Yu.N.Ovchinnikov and I.M.Segal, Ann.Phys.(NY) 123 (1979) 274

5)B.G.Giraud and Y.Hahn, Nucl.Phys. A588 (1995) 653

6)J.Gilewicz, Approximants de Padé, A.Dold and B.Eckmann eds. (Springer 1978, Berlin, Heidelberg, New-York) pp.264-265 
It is equivalent to find eigenstates $\mid \psi>$ from the condition

$$
\left(\omega^{2}+p^{2}-\frac{\mathcal{V}^{\prime}}{}\right) \mid \psi>=0
$$

or eigenstates $\left.\right|^{\prime}>=W^{\prime} \mid \psi>$ from the condition

$$
\left.\left[-W^{\prime}\left(\omega^{2}+p^{2}\right)^{-1} W^{\prime}\right]\right|^{\prime}>=0
$$

with $\mathcal{V}^{\prime}=\left[W^{\prime}\right]^{2}$. From Eq.(A1), the eigenvalue $-\omega^{2}$ and the coupling constant $-1 /$ are related according to

$$
-2 \omega^{2} \frac{d \omega}{d}=\frac{<\psi\left|\mathcal{V}^{\prime}\right| \psi>}{<\psi \mid \psi>}
$$

where for convenience the norm $\langle\psi \mid \psi\rangle$ is written explicitly.

We are considering $s$-wave eigenstates for scalar potentials, and furthermore, for simplicity, our local potentials $\mathcal{V}^{\prime}$ are assumed to strictly vanish beyond some radius $r_{0}$, while being strictly positive inside $r_{0}$. Square wells are just a special case. For our argument, $\psi$ is now normalized according to

$$
\psi(\vec{r})=\frac{\exp \left[-\omega\left(r-r_{0}\right)\right]}{r}, \quad r>r_{0}
$$

namely $\psi=1 / r_{0}$ at the edge of the potential. Then the total norm is

$$
<\left.\psi\left|\psi>=4 \pi \int_{0}^{r_{0}} r^{2} d r\right| \psi(r)\right|^{2}+\frac{1}{2 \omega} .
$$

For a square well, it is trivial to show that the inner term, $\int_{0}^{r_{0}}$, remains finite when $\omega \rightarrow 0$. The same is easy to generalize for a large class of potentials. Hence $\langle\psi \mid \psi\rangle$ diverges like $1 / \omega$. Simultaneously, $\left\langle\psi\left|\mathcal{V}^{\prime}\right| \psi\right\rangle$, an inner integral again, remains finite, with a finite, non vanishing limit when $\omega \rightarrow 0$. (This limit compensates the kinetic energy, since the binding vanishes.) Hence, finally, $\left\langle\psi\left|\mathcal{V}^{\prime}\right| \psi\right\rangle /(\omega<\psi \mid \psi>)$ has a finite limit. Since, at the same time, reaches the reciprocal of the "critical" strength $\lambda^{\prime}$, we can conclude from Eq.(A3) that $d / d \omega$ has a finite, non vanishing, negative limit $-\sigma_{0}$. 